\title{
CORRECTIONS
}

\section{Management of chronic epilepsy}

We would like to make a clarification to this Clinical Review (BMJ 2012;345:e4576, doi:10.1136/bmj.e4576) regarding the table "NICE guidelines on antiepileptic drug treatment." The drugs carbamazepine and oxcarbazepine appear twice for the seizure type "Generalised tonic-clonic" under the columns for both "First line treatment" and "Do not consider (may worsen seizures)." This recommendation is in line with NICE guidelines and is not an error. Carbamazepine and oxcarbazepine are recommended as first line treatment for generalised tonic-clonic seizures. However, in some specific generalised epilepsy syndromes, which may have generalised tonic-clonic seizures as part of the presentation, the use of these drugs can aggravate the condition with worsening seizures.

Cite this as: BMJ 2012;345:e5535

๑ BMJ Publishing Group Ltd 2012 